\title{
Waves in Poynting-flux dominated jets
}

\author{
John G. Kirk and Iwona Mochol \\ Max-Planck-Institut für Kernphysik, Postfach 1039 80, 69029 Heidelberg, Germany \\ email: john.kirk@mpi-hd.mpg.de, iwona.mochol@mpi-hd.mpg.de
}

\begin{abstract}
High-energy emission from blazars is thought to arise in a relativistic jet launched by a supermassive black hole. The rapid variability of the emission suggests that structure of length scale smaller than the gravitational radius of the central black hole is imprinted on the jet as it is launched, and modulates the radiation released after it has been accelerated to high Lorentz factor. We describe a mechanism which can account for the acceleration of the jet, and for the rapid variability of the radiation, based on the propagation characteristics of nonlinear waves in charge-starved, polar jets. These exhibit a delayed acceleration phase, that kicks-in when the inertia associated with the wave currents becomes important. The time structure imprinted on the jet at launch modulates the photons produced by the accelerating jet provided that the electromagnetic cascade in the black-hole magnetosphere is not prolific.
\end{abstract}

Keywords. MHD - plasmas - waves - BL Lacertae objects: general

\section{Introduction}

The blazar PKS 2155-304 has been observed to exhibit remarkably rapid variability at very high flux levels (Aharonian et al. 2007, HESS Collaboration 2010). In the most extreme flare, structure in the jet is implied that is roughly one hundred times smaller than the gravitational radius $r_{\mathrm{g}}=G M / c^{2}$, with $M$ the black-hole mass (Begelman et al. 2008). This structure is presumably imprinted as the jet leaves the black-hole magnetosphere, suggesting that the axisymmetric, ideal MHD approximation might not provide an adequate description. In analogy with pulsar magnetospheres (Spitkovsky 2006) it seems reasonably to assume that a generalised form of the axisymmetric Blandford-Znajek mechanism (Blandford \& Znajek 1977) causes the non-axisymmetric magnetosphere of a rotating black hole to drive a Poynting-flux dominated jet, part of which might propagate in the low-density funnel around the rotation axis. In the following, we develop this idea by examining the propagation characteristics of nonlinear electromagnetic waves above the polar regions of a rotating black hole.

\section{Jet parameters}

Matter accreting onto a rotating black hole fails to penetrate into a conical region or "funnel" around the rotation axis (De Villiers \& Hawley 2003; McKinney \& Gammie 2004), where the density is presumably dominated by an electron-positron plasma produced in an electromagnetic cascade. The physical conditions in this outflowing plasma can be described by three dimensionless parameters: (i) the nonlinearity or strength parameter $a=a_{0}(c / \omega r)$, where $\omega / 2 \pi$ is the wave frequency and

$$
a_{0}=\left[4 \pi e^{2} L /\left(m^{2} c^{5} \Omega_{\mathrm{s}}\right)\right]^{1 / 2}=3.4 \times 10^{14} L_{46}^{1 / 2}
$$

( $L$ is the luminosity in solid angle $\Omega_{\mathrm{s}}, L_{46}$ the " $4 \pi$ " luminosity in units of $10^{46} \mathrm{erg} / \mathrm{s}$, which, for PKS 2155-304 is approximately unity), (ii) the mass-loading of the wind $\mu=$ $L / \dot{M} c^{2}$ (Michel 1969), with $\dot{M}$ the mass flux in the jet, (iii) the magnetisation parameter 
$\sigma$ which is the ratio of the energy flux carried by electromagnetic fields to that carried by particles. For monoenergetic electrons and positrons of Lorentz factor $\gamma$,

$$
\sigma=(\mu / \gamma)-1
$$

We specify its value at the "launching" radius, inside of which the ideal MHD approximation is assumed to hold, and denote this (constant) quantity by $\sigma_{0}$. The particle Lorentz factor at the launching point is then $\gamma_{0}=\mu /\left(\sigma_{0}+1\right)$. An alternative, more intuitive measure of the mass-loading, is the pair multiplicity $\kappa$, defined as in pulsar physics (e.g., Lyubarsky \& Kirk 2001). Here, we specify $\kappa$ by its value $\kappa_{r_{\mathrm{g}}}$ at $r=r_{\mathrm{g}}$ :

$$
\kappa_{r_{\mathrm{g}}} \approx \frac{a_{0}}{4 \mu}\left(\frac{c}{\omega r_{\mathrm{g}}}\right)
$$

\section{The two-fluid model}

We adopt the model of two cold, charged fluids $\left(e^{ \pm}\right)$in a Kerr metric, following Khanna (1998). To keep the analysis tractable, only transverse waves are treated. We also assume radial propagation, circular polarisation and charge neutrality. It follows that the fluids have the same radial component of the four-velocity, which we write as a dimensionless momentum $p_{\|}$. The transverse components of the fluid momenta are equal and opposite and are denoted by $\pm p_{\perp}$. The evolution is described by the continuity equation, the equations of motion of the fluids and the two relevant Maxwell equations (Faraday's law and Ampère's law) in the small-wavelength approximation, $c / \omega r \sim \epsilon \ll 1$. Although smaller, we assume that the wavelength is of the same order in $\epsilon$ as the gravitational radius $r_{g}$. Under these conditions general relativistic effects do not appear in the governing equations. In the lowest-order system of equations, we search for large-amplitude plane-wave solutions using the approach introduced by Akhiezer \& Polovin (1956).

\subsection{Plane waves}

Expressing all wave quantities in terms of the phase $\phi$ enables the fluid equations to be converted into a set of ordinary differential equations, which can be integrated to find nonlinear wave solutions. Concentrating on waves with subluminal phase velocity, $\beta_{\mathrm{w}}<1$, one finds that the particles are in resonance with the wave in the sense that the radial components of the fluid velocities equal the phase velocity of the radially propagating wave. In this case the plasma current is directed along the magnetic field and, to lowest order, the forces exerted on the fluids by the fields vanish. Viewed from a frame that moves radially with speed $\beta_{\mathrm{w}}$, the electric field vanishes, and the wave is simply a static magnetic field of constant magnitude whose direction rotates through $2 \pi$ radians over one wavelength. The rate at which the $B$-vector rotates is arbitrary, being determined by the dependence of the fluid proper density $n$ on phase. In the following, we select the simplest case, where $n,|B|^{2}$ and $\left|p_{\perp}\right|^{2}$ are all constant and the wave is a monochromatic magnetic shear, in rotating (complex) coordinates: $B \propto p_{\perp} \propto \mathrm{e}^{ \pm i \phi}$.

\subsection{Radial evolution}

The radial dependence of the wave goes through three phases. Defining the dimensionless radius $R=\mu \omega r /\left(a_{0} c\right)$, on finds that $\left|p_{\perp}\right| \ll 1$ when $R \ll \mu / \sigma_{0}$, and the wave is essentially a cold MHD structure in which the inertia associated with the current is negligible. There is no acceleration of either the wave speed or the fluids in this regime, and the magnetisation parameter $\sigma$ remains constant at its initial value. At intermediate radii, 


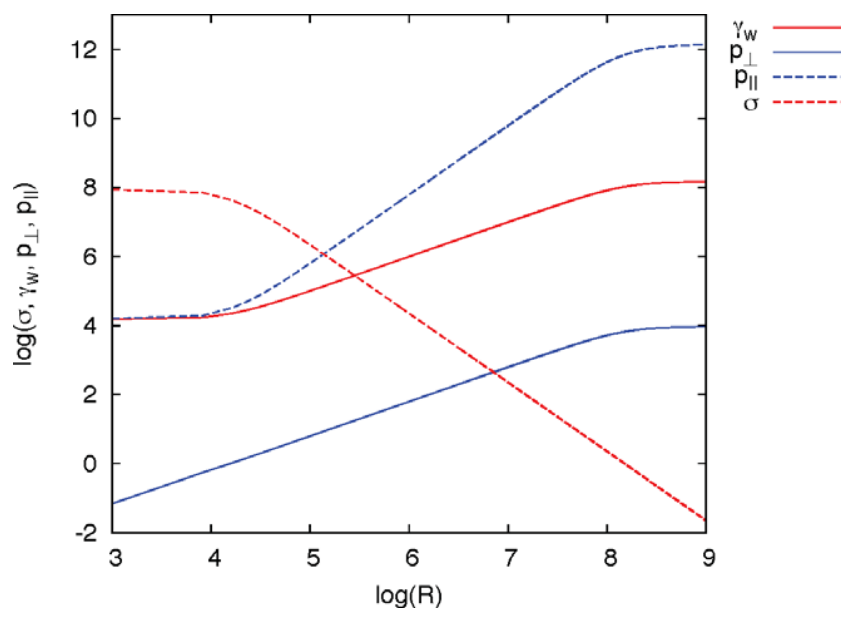

Figure 1. The magnetisation parameter $\sigma$, radial and transverse fluid momenta $p_{\|}$and $\left|p_{\perp}\right|$ and Lorentz factor of the wave, $\gamma_{\mathrm{w}}$, as functions of the dimensionless radius $R=\mu r \omega /\left(a_{0} c\right)$, for $\sigma_{0}=10^{8}$ and $\mu=1.4 \times 10^{12}$.

where $\left|p_{\perp}\right| \gg 1$, and $\sigma \gg 1$, one readily finds an approximate solution, provided the wave is launched not too close to the sound speed (i.e., for $\gamma_{0} \gg \sigma_{0}^{1 / 2} \gg 1$ ):

$$
\begin{aligned}
\gamma_{\mathrm{w}} & \approx R & \gamma & \approx R^{2} \sigma_{0} / \mu \\
\sigma & \approx \mu^{2} /\left(R^{2} \sigma_{0}\right) & \left|p_{\perp}\right| & \approx R \sigma_{0} / \mu .
\end{aligned}
$$

This solution is valid until $\sigma$ becomes of the order of unity, which occurs at $R \approx \mu / \sqrt{\sigma_{0}}$ Finally, at large radius, $R \gg \mu / \sqrt{\sigma_{0}}$, only kinetic energy remains: $\gamma \approx \mu, \gamma_{\mathrm{w}} \approx \mu / \sqrt{\sigma_{0}}$, $\left|p_{\perp}\right| \approx \sqrt{\sigma_{0}}$. This behaviour is illustrated in Fig. 1, which plots the solutions for values of interest in the case of PKS 2155-304: $\sigma_{0}=10^{8}, \mu=1.4 \times 10^{12}$.

\section{Application to blazar variability}

The radius $r_{\text {acc }}$ at which acceleration begins, corresponding to $R \approx \mu / \sigma_{0}$, is

$$
r_{\mathrm{acc}} \approx r_{\mathrm{g}} a_{0}^{1 / 3} \kappa_{r_{\mathrm{g}}}^{2 / 3}\left(\omega r_{\mathrm{g}} / c\right)^{-1 / 3}=1.2 \Delta t_{100}^{1 / 3} \kappa_{r_{\mathrm{g}}}^{2 / 3} L_{46}^{1 / 6} M_{9}^{2 / 3} \mathrm{pc},
$$

where the variation timescale in units of $100 \mathrm{~s}$ is $\Delta t_{100}=(2 \pi / \omega) /(100 \mathrm{~s})$ and $M_{9}=$ $M / 10^{9} \mathrm{M}_{\odot}$. We analyse the case of mildly supersonic launch: $\sigma_{0} \approx \mu^{2 / 3}$, so that

$$
\gamma_{0}=6.5 \times 10^{3} \Delta t_{100}^{1 / 3} \kappa_{r_{\mathrm{g}}}^{-1 / 3} L_{46}^{1 / 6} M_{9}^{-1 / 3}
$$

The solutions presented in section 3 eventually convert all of the Poynting flux to kinetic energy flux at large radius. However, in the case of blazars, this is unlikely to be realised, since the resulting Lorentz factor $(=\mu)$ is very large. Instead, dissipative processes, which we have so far neglected, are likely to intervene. These include (i) instabilities in the wave-solution, which can lead to an effective friction between the fluids, equivalent to a finite resistivity in MHD language, (ii) interaction of the jet with the external medium, causing collimation, or matter entrainment, and (iii) interaction with ambient photons, causing jet deceleration and giving rise to inverse Compton photons.

If jet radiation is modulated at the wave frequency, it is straightforward to derive a criterion on the size of the emitting region (assumed $\sim r$ ) and the Lorentz factor of the jet such that the modulations are not washed out by the travel time of the signal across the source (Michel 1971, Arons 1979, Kirk et al. 2002): $\gamma_{\mathrm{w}}^{2} 2 \pi c / \omega>r$. Since 
$\gamma_{\mathrm{w}} \propto r$ in the acceleration region, this condition is fulfilled everywhere, provided it is satisfied at $r=r_{\text {acc }}$, where $\gamma_{\mathrm{w}}=\gamma_{0}$. The requirement that modulation on a timescale of $100 \Delta t_{100}$ seconds should not be filtered out leads, from (4.1) and (4.2), to an upper limit on the multiplicity:

$$
\kappa_{r_{\mathrm{g}}}<14 \Delta t_{100} L_{46}^{1 / 8} M_{9}^{-1}
$$

Equation (4.3) implies that those sources in which conditions close to the black hole might favour the development of prolific cascades — such as strong disk emission should not exhibit extreme variability. In the absence of such a cascade, the magnetosphere is able to support vacuum gaps. These are thought to produce highly nonstationary discharges in the pulsar environment (Levinson et al 2005, Timokhin 2010). Thus, if our model is correct, the rapid variations of the TeV gamma-ray flux of a blazar could be a direct manifestation of the fluctuating discharge of a vacuum gap in the black-hole magnetosphere.

\section{Summary and conclusions}

Circularly polarized, large-amplitude waves are likely to be generated in the polar-cap regions of a rotating black hole endowed with an external magnetic field. These regions are believed to be charge-starved, so that the ideal MHD description might fail because the inertia of the current carriers cannot be neglected. In the case of the subluminal magnetic shear which we analyse above, this happens when $p_{\perp}$ becomes relativistic, and it causes the wave and jet to accelerate. Additional effects, such as collimation or interaction with an external radiation field may enhance the acceleration process. However, a detailed investigation remains to be done.

We do not address the question of the mechanism of $\gamma$-ray production in the accelerating jet. However, if, as seems inevitable, the emission is modulated at the wave frequency, we show that the large spatial extent of the emission region fails to wash out this signal, provided that the density of pairs injected in the magnetosphere by an electromagnetic cascade is sufficiently small.

\section{References}

Aharonian, F. et al. 2007, ApJ, 664, L71

Akhiezer, A. I. \& Polovin, R. V. 1956, Sov. Phys. JETP, 3, 696

Arons, J. 1979, Space Sci. Rev., 24, 437

Begelman, M. C., Fabian, A. C., \& Rees, M. J. 2008, MNRAS, 384, L19

Blandford, R. D. \& Znajek, R. L. 1977, MNRAS, 179, 433

De Villiers, J. \& Hawley, J. F. 2003, ApJ, 592, 1060

HESS Collaboration, Abramowski, A. et al. 2010, arXiv:1005.3702

Khanna, R. 1998, MNRAS, 294, 673

Kirk, J. G., Skjæraasen, O., \& Gallant, Y. A. 2002, A\&A, 388, L29

Levinson, A., Melrose, Judge, D. A., \& Luo, Q. 2005, ApJ, 631, 456

Lyubarsky, Y. \& Kirk, J. G. 2001, ApJ, 547, 437

McKinney, J. C. \& Gammie, C. F. 2004, ApJ, 611, 977

Medin, Z. \& Lai, D. 2010, MNRAS, 406, 1379

Michel, F. C. 1969, ApJ, 158, 727

Michel, F. C. 1971, Comments on Astrophysics and Space Physics, 3, 80

Spitkovsky, A. 2006, ApJ, 648:L51-L54

Timokhin, A. N. 2010, arXiv:1006.2384 


\section{Discussion}

\section{PE'ER:}

(a) Is there any evidence of an annihilation line?

(b) Are you considering any protons that can carry kinetic energy and momentum?

KIRK:

(a) Not to my knowledge. I also would not expect a signature, because the pair density in the jet is very low.

(b) I have assumed a pair plasma. If protons are present (perhaps introduced into the funnel by decaying neutrons) the structure of the nonlinear wave modes is more complicated, but could, in principle, be analysed using the same approach.

LEVINSON: How is it possible to have variations on a time scale shorter than $r_{\mathrm{g}} / c$ ?

KIRK: I don't think there is any fundamental reason for excluding structure in a nonaxisymmetric magnetic field on scales less than $r_{\mathrm{g}}$, at a position close to $r=r_{\mathrm{g}}$. I just assume these are transported radially to large distance. The transverse length scale is stretched, but the radial length scale remains fixed in the lab. frame for a relativistic flow. Provided the Lorentz factor is large, the photons that are produced at large radius carry the information on the radial length scale to the observer, and this is not smeared out by the large transverse size of the source. 